\title{
Targeting the Vascular Endothelium - Novel Approaches
}

\author{
Dario Neri \\ Department of Chemistry and Applied Biosciences, Swiss Federal Institute of Technology, Zurich, Switzerland
}

Angiogenesis, i.e., the proliferation of new blood vessels from pre-existing ones, is an underlying process in many human diseases, including cancer, blinding ocular disorders and rheumatoid arthritis. The ability to selectively target and occlude neovasculature will be potentially useful in diagnosis and treatment of angiogenesis-related diseases.

A good-quality marker for both tumoural and nontumoural neovasculature is the extra-domain B (ED-B) of fibronectin, a sequence of 91 aminoacids that can be inserted into the fibronectin molecule by a mechanism of alternative splicing.

To date, the production of monoclonal antibodies directly recognising the ED-B domain has not been possible using hybridoma technology, because of tolerance. In collaboration with Prof. Dr. Luciano Zardi (Istituto Nazionale per la Ricerca sul Cancro, Genova, Italy), we have overcome this problem using large synthetic antibody phage libraries, generating several high-affinity antibody fragments specific for fibronectin containing the ED-B domain, and mapping their epitope onto the three-dimensional structure of the antigen. These antibodies stain vascular structures in tumour sections and selectively target tumour neovasculature, as shown in tumour-bearing mice using infrared fluorescence and radioactive techniques. Increased binding affinity leads to improved targeting of tumoural angiogenesis, as demonstrated by biodistributions studies performed using the L19 antibody fragment with affinity for the ED-B domain in the picomolar range and L19 mutants with reduced affinity.

The ability of radiolabelled L19 to target tumours in patients with cancer has recently been demonstrated using scintigraphic detection methods. A number of derivatives of scFv(L19), with a therapeutic potential, have been studied in animal models. The antibody, coupled to a photosensitizer, was shown to be able to mediate a complete and selective photo-occlusion of new blood vessels in a rabbit model of ocular angiogenesis. Indeed, the EDB domain of fibronectin is strongly expressed in angiogenesis-related ocular disorders. A fusion of $\operatorname{scFv}(\mathrm{L} 19)$ with truncated tissue factor (a pro-coagulant protein) mediated the rapid intraluminal blood coagulation in tumour blood vessels, but not in normal blood vessels. As a result, a dramatic collapse of the tumour mass could be observed, particularly for large tumour masses.

Systemic administration of cytokines, such as IL-2, TNF, GM-CSF or IL-12 can render some non-immunogenic tumours immunogenic, activating a protective immunity. However, systemic administration of cytokines is often associated with severe toxic side-effects, preventing the administration of a curative dose. A possible way of increasing the therapeutic index of cytokines consists of fusing them to antibodies, which mediate a preferential accumulation of the cytokine at the tumour site. Indeed, in the past decade, several groups have reported different antibody-cytokine fusions for different tumour associated

\begin{tabular}{ll}
\hline KARGER & ( ) 2003 S. Karger AG, Basel \\
1424-8832/03/0337-0007\$19.50/0 \\
$\begin{array}{l}\text { Fax +4161306 1234 } \\
\text { E-Mail karger@karger.ch } \\
\text { www.karger.com }\end{array}$ & $\begin{array}{l}\text { Accessible online at: } \\
\text { www.karger.com/journals/pht }\end{array}$
\end{tabular}

Dario Neri, Professor of Biomacromolecules

Department of Chemistry and Applied Biosciences

Swiss Federal Institute of Technology

Irchel Y36 M14, Winterthurerstr. 190, CH-8057 Zurich (Switzerland)

Tel. +4116356063, Fax +4116356886

E-Mail neri@pharma.ethz.ch 
antigens. $\operatorname{ScFv}(\mathrm{L} 19)$ has been expressed as fusion protein with potently immunostimulatory cytokines (such as IL-2, IL-12, TNF). These novel proteins were shown to retain both antibody and cytokine functions and to show superior anti-cancer activities, as compared to equivalent amounts of free cytokine (and antibody).
The results obtained are of therapeutic relevance, since the ED-B domain of fibronectin, a naturally-occurring marker of angiogenesis identical in mouse and man, is expressed in the majority of aggressive solid tumours, but is undetectable in normal vessels and tissues.

\section{References}

Birchler M, Viti F, Zardi L, Spiess B, Neri D: Selective targeting and photocoagulation of ocular angiogenesis mediated by a phage-derived recombinant antibody. Nature Biotechnol 1999; 17:984-988.

Carnemolla B, Borsi L, Balza E, Castellani P, Meazza R, Berndt A, Ferrini S, Kosmehl H, Neri D, Zardi L: Enhancement of the anti-tumour properties of interleukin-2 by its targeted delivery to the tumour blood vessel extracellular matrix. Blood 2002;99: 1659-1665.
Halin C, Rondini S, Nilsson F, Berndt A, Kosmehl H, Zardi L, Neri D: Enhancement of the anti-tumour activity of interleukin-12 by targeted delivery to neo-vasculature. Nature Biotechnol 2002;20: 264-269.
Santimaria M, Moscatelli G, Giovannoni L, Leprini A, Neri G, Zardi L, Neri D, Riva P: Immunoscintigraphic detection of the ED-B domain of fibronectin, a marker of angiogenesis, in patients with cancer. Clin Cancer Res 2003;9:741-749. 